\title{
CREBBP is a Novel Biomarker for Diagnostic and Prognostic of Patients with Renal Cell
}

\section{Carcinoma}

\author{
Pei Chen', Qiqi Zhang², Yuanguo Li ${ }^{3}$, Fangxu Li ${ }^{4}$, Tian Qin ${ }^{2,3}$, Jing Qi ${ }^{2,3}$, Tiecheng Wang ${ }^{3 *}$ and Chaoxiang Lv ${ }^{2,3 *}$ \\ ${ }^{1}$ School of Medicine, Jianghan University, Wuhan 430058, \\ P.R. China. \\ ${ }^{2}$ The Key Laboratory of Molecular Epigenetics of MOE, \\ Institute of Genetics and Cytology, Northeast Normal \\ University, Changchun, Jilin 130021, P.R. China. \\ "Corresponding author \\ Chaoxiang Lv and Tiecheng Wang, The Key Laboratory of Molecular \\ Epigenetics of MOE, Institute of Genetics and Cytology, Northeast Normal \\ University, Changchun, Jilin 130021, P.R. China, Changchun Veterinary \\ Research Institute, Chinese Academy of Agricultural Sciences. Changchun \\ 130122, PR China.
}

${ }^{3}$ Changchun Veterinary Research Institute, Chinese Academy of Agricultural Sciences. Changchun 130122, PR China.

Submitted: 05 May 2021; Accepted: 12 May 2021; Published: 20 May 2021

${ }^{4}$ College of life sciences, Shandong Normal University, Jinan 250014, P.R. China.

Citation: Pei Chen, Qiqi Zhang, Yuanguo Li, Fangxu Li, Tian Qin, et al. (2021) CREBBP is a Novel Biomarker for Diagnostic and Prognostic of Patients with Renal Cell Carcinoma. Medical \& Clinical Research 6(5): 527-534.

\begin{abstract}
Annotation
As an important transcription co-regulator, CREBBP played important roles in gene transcription and signal transduction. It had been reported that its low expression was closely related to cancer progression. We explored the roles of CREBBP expression among renal cell carcinoma in order to clarify its potential clinical application value in the diagnosis, pathological process and patients prognosis. In the present study, we found that CREBBP expression was reduced in renal cell carcinoma compared with normal tissues, and its expression was negatively correlated with tumor progression. Furthermore, lower $C R E B B P$ expression in dead than survival patients, and it had good clinical diagnostic value in renal cell carcinoma. The OS and RFS of patients with low CREBBP expression were significantly shortened, and it was a dependent predictor for poor prognosis. Additionally, we also found the reactive oxygen species pathway, DNA repair and myc-targets $V 2$ signaling were significantly enriched in the low CREBBP expression phenotype. In conclusion, low CREBBP expression could be regarded as a prognosis biomarker and potential therapeutic target for renal cell carcinoma.
\end{abstract}

Keywords: Renal Cell Carcinoma, Histone Acetylation, CREBBP, Molecular Markers, Prognosis

\section{Introduction}

Renal cell carcinoma is a malignant tumor of the urinary system, and its morbidity and fatality rate are steadily increasing $[1,2]$. The disease not only damaged the urinary system, but also caused malignant metastasis, which endangered the lives of patients [3, 4]. Most patients are already in the advanced stage when they are diagnosed and cannot receive radical surgery, which has seriously affected human health. Although the great improvements in diagnosis and treatment of renal cell carcinoma had been achieved, the 5-year survival rate of patients is still frustrating, and the prognosis is disappointing [5]. Thus, it is still extremely important to find useful biomarkers for diagnosis and prognosis of renal cell carcinoma.

The acetylation of histone is a reversible epigenetic modification, and the most obvious feature is lysine acetylation [6]. It had been involved in a variety of biological processes, such as cell cycle, signaling transduction and gene expression [7-9]. The disorder of the modification system will contribute to research the etiology of human diseases, such as cancer [10]. Indeed, the abnormality of this system is closely related to the progression of a variety of human malignancies, including esophageal cancer, colorectal cancer, gastric cancer and liver cancer [11-14]. These findings suggested that the enzymes of regulation histone acetylation might become a symbol of tumor prognosis and a new target of antitumor therapy.

The cAMP responsive element binding protein (CREBBP), originally isolated from nuclear proteins, was involved in the transcriptional co-activation of multiple transcription factors [1517]. This gene had important roles in embryonic development, cell growth and homeostasis by combining chromatin remodeling with transcription factor recognition $[18,19]$. It had been reported that the protein encoded by CREBBP regulated chromatin 
remodeling through inducing histone acetylation to promote the growth of malignant cells [20]. In addition, CREBBP mutations not only caused Rubinstein-Telby syndrome (RTS) and acute myeloid leukemia, but also promoted tumor cell differentiation and metastasis [21]. These findings indicated that the expression of CREBBP might have potential clinical evaluation prospects. Recently, in colorectal cancer, the expression of CREBBP was related to the type and grade of tumor [22]. Its mutation was an independent prognostic factor for diffuse large B-cell lymphoma [23]. However, the role of its expression in the clinical diagnosis, treatment and prognosis of renal cell carcinoma was rarely reported.

Here, we explored the effect of CREBBP expression on clinical characteristics in patients with renal cell carcinoma. By using bioinformatics tools, we revealed the important role of CREBBP expression in clinical diagnosis and prognostic evaluation. In addition, we also analyzed the association between CREBBP expression and patients' overall survival (OS) and relapsefree survival (RFS). Our findings suggested that low CREBBP expression was a risk factor for poor survival, and revealed that its expression may be an unexpected role for the diagnosis and prognosis of renal cell carcinoma.

\section{Materials and Methods}

\section{The data-set of clinical mining}

Based on the The-Cancer-Genome-Atlas (TCGA) dataset, we collected 534 patients with KIRC of the clinical information and the RNAseq value of CREBBP. Subsequently, we converted the RNAseq value into RSEM by estimating normalized counts as $\log 2(\mathrm{x}+1)$ [24]. All data were analyzed by selecting $\mathrm{R}$ software (version 4.0.1).

\section{Gene-Set-Enrichment-Analysis (GSEA)}

In order to explore the underlying mechanism of CREBBP expression on the prognosis of KIRC, we selected GSEA (version 4.0.3). The gene-sets database of "h.all.v7.2.symbols.gmt" was performed to determine the differences in phenotypes of different genes [25]. P-value and false-discovery-rate (FDR) were used to determine significant differences between groups $(\mathrm{P}<0.05$, FDR $<0.25$ ).

\section{Statistical Analysis}

Ggplot2 in R software was used to display the expression of CREBBP in the clinical data-set of KIRC patients, and the correlation between its expression and the clinical characteristics of the patients was evaluated by the chi-square test. The pROC software package was used to evaluate the diagnostic significance of CREBBP expression [26]. Survival package and risk regression model analyzed the impact of CREBBP expression on survival rate and poor prognosis of patients. Statistical difference was regarded as $\mathrm{P}<0.05$.

\section{Results}

The Clinical Characteristics of Patients and The Expression Pattern CREBBP in KIRC To analyze the relationship between CREBBP expression and clinical characteristics of patients, we obtained the clinical data-sets from the TCGA database, including the patient's age, gender, histological grade and histological stage, as well as M/N/T classification, vital status and relapse (Table S1). In addition, we also analyzed the expression pattern of CREBBP. Compared with normal tissues, its expression was significantly reduced in tumor tissues $(\mathrm{P}=1.55 \times 10-5$; Figure 1$)$. Further analysis revealed that the expression of $\mathrm{CREBBP}$ had significant difference at the histological level $(\mathrm{P}=4.21 \times 10-5)$ and the pathological stage $(\mathrm{P}=6.82 \times 10)-4)$, T classification $(\mathrm{P}=0.00460), \mathrm{M}$ classification $(P=0.00560)$ and vital status $(P=4.77 \times 10-6)$. These findings indicated that CREBBP was down-regulated in KIRC, and it was likely to be closely related to tumor progression and the survival status of patients.
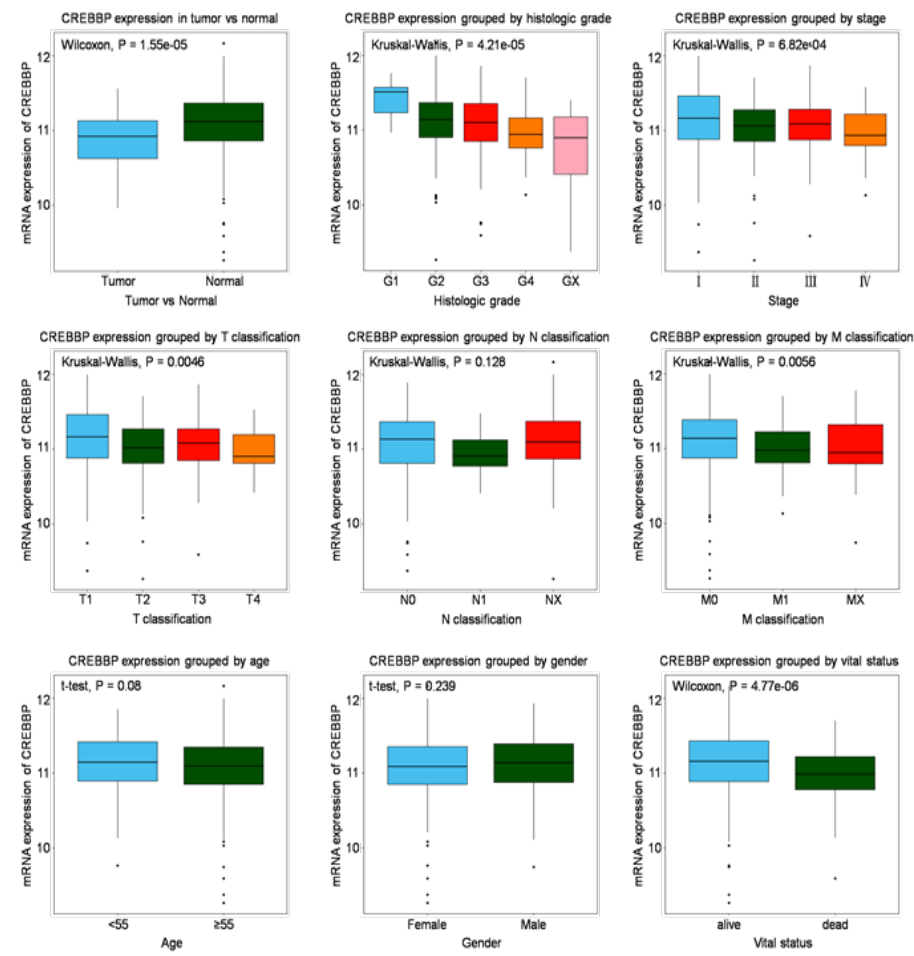

Figure 1: The Expression pattern of CREBBP in KIRC.

The expression of CREBBP between healthy individual and tumor patients was compared. The expression of CREBBP was compared in different sub-groups, including patient's histologic grade, stage and $\mathrm{T} / \mathrm{N} / \mathrm{M}$ classification, as well as age, gender and vital status.

The Effect of CREBBP Expression on Diagnostic Value of KIRC Evaluation of the diagnostic value of a biomarker was the basis of clinical practice and treatment options [27]. Thus, we constructed ROC curve to compare the contribution of CREBBP expression between healthy individuals and patients with KIRC. The shaded part of the curve represented diagnostic value. We found that CREBBP expression had good diagnostic value $(\mathrm{AUC}=0.657$; Figure 2). In addition, we also evaluated its diagnostic value of in different stages of KIRC, including stage I (AUC $=0.621)$, stage II $(\mathrm{AUC}=0.694)$, stage III $(\mathrm{AUC}=0.651)$ and stage IV $(\mathrm{AUC}=$ $0.568)$. 

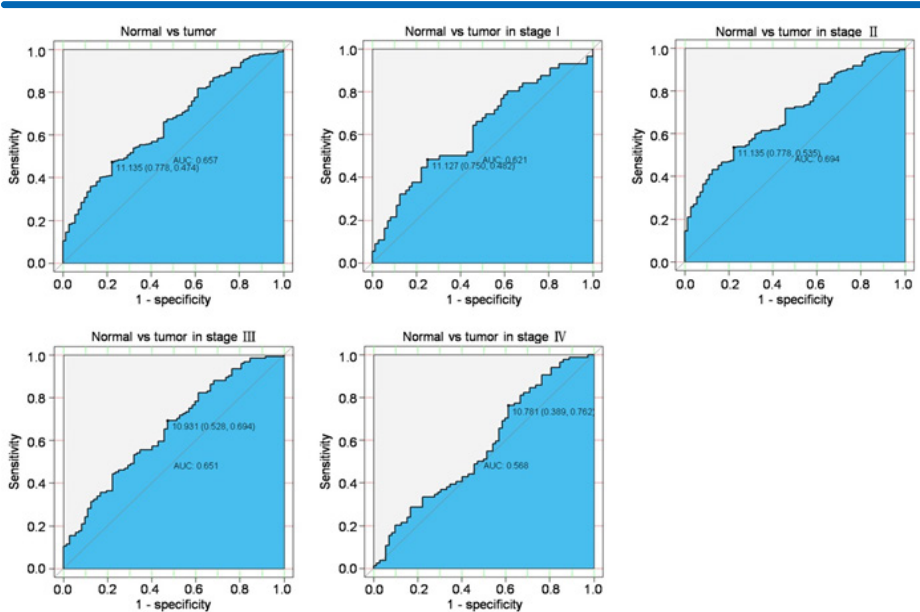

Figure 2: Diagnosis value of CREBBP expression in KIRC.

The ROC curves of CREBBP expression was generated in cancerous vs. normal tissues, and in different stages of KIRC.

The Correlation Between CREBBP Expression and Clinical Characteristics of Patients with KIRC

We previously showed that CREBBP expression had good diagnostic value in KIRC. In order to further analyze the relationship between its expression and the clinical characteristics of patients, we divided patients into high expression and low expression groups according to the ROC curve threshold. On this basis, chi-square test was implemented (Table S2). We found that low CREBBP expression was significantly related to the patient's histologic grade $(\mathrm{p}=0.001)$, pathologic stage $(\mathrm{p}=0.010)$, $M$ classification $(\mathrm{p}=0.014)$, T classification $(\mathrm{p}=0.027)$ and survival status $(\mathrm{p}=0.0001)$.

\section{The Effect of low CREBBP Expression on OS of Patients with KIRC}

To further evaluate the impact of CREBBP expression on the OS of patients, Kaplan-Meier curves were established. We found that OS was significantly shortened in patients with low CREBBP expression ( $\mathrm{p}=0.0012$, Figure 3$)$. Sub-groups analysis found that low CREBBP expression was positively correlated with poor OS in female patients $(\mathrm{P}=0.00055)$. This phenomenon also applied to G3 / G4 / GX ( $\mathrm{P}=0.006)$, Stage III/IV ( $\mathrm{P}=0.013), \mathrm{T} 3(\mathrm{P}=0.020)$, N0 $(\mathrm{P}=0.070), \mathrm{N} 1 / \mathrm{NX}(\mathrm{P}=0.0074)$ and $\mathrm{M} 0(\mathrm{P}=0.027)$. In addition, we selected the difference variables in univariate analysis for multivariate analysis by the establishment of a risk regression model. As shown in Table 1, low CREBBP expression predicted poor OS of patients with KIRC $(\mathrm{p}=0.026)$.

Table 1: Univariate and Multivariate analysis of Over Survival in patients with KIRC.

\begin{tabular}{|l|c|c|c|c|c|c|}
\hline & \multicolumn{3}{|c|}{ Univariate analysis } & \multicolumn{3}{c|}{ Multivariate analysis } \\
\hline & Hazard Ratio & CI95 & Pvalue & $\begin{array}{c}\text { H a z a r d } \\
\text { Ratio }\end{array}$ & CI95 & Pvalue \\
\hline Age & 1.92 & $1.31-2.82$ & 0.001 & 1.64 & $1.11-2.44$ & 0.014 \\
\hline Gender & 1.04 & $0.75-1.43$ & 0.826 & & & \\
\hline Histologic grade & 1.02 & $0.89-1.12$ & 0.999 & & & \\
\hline Pathologic stage & 1.96 & $1.71-2.24$ & 0.001 & 2.00 & $0.43-2.59$ & 0.001 \\
\hline M classification & 2.49 & $1.94-3.19$ & 0.001 & 0.98 & $0.61-1.62$ & 0.939 \\
\hline N classification & 0.86 & $10.74-1.01$ & 0.063 & & & \\
\hline T classification & 2.07 & $1.74-2.46$ & 0.001 & 0.91 & $0.62-1.35$ & 0.655 \\
\hline CREBBP & 1.71 & $1.23-2.36$ & 0.001 & 1.46 & $1.05-2.03$ & 0.026 \\
\hline
\end{tabular}



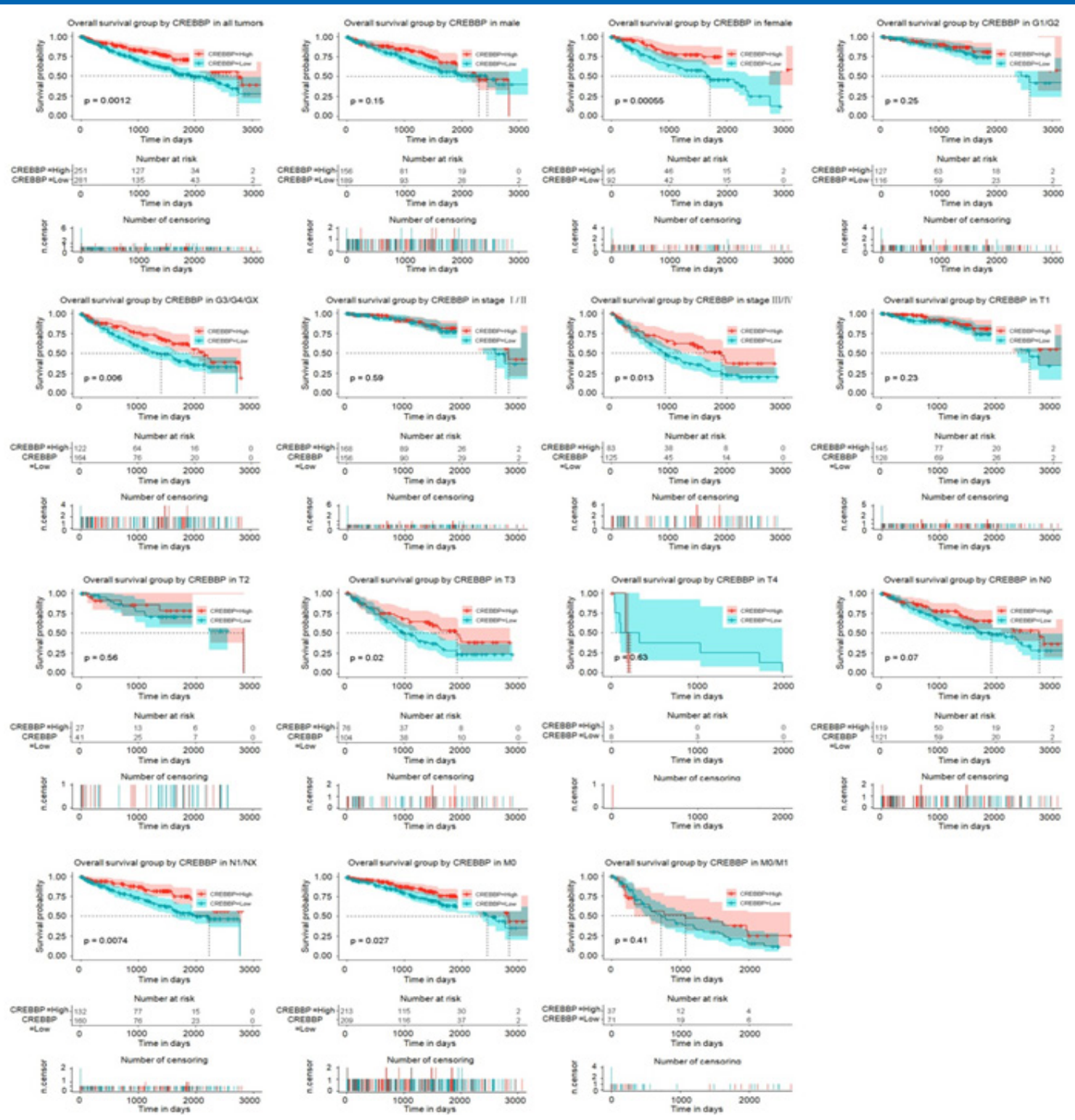

Figure 3: Effect of CREBBP Expression on OS in KIRC.

Kaplan-Meier curves of CREBBP expression in all patients and in subgroup.

\section{The Effect of Low CREBBP Expression on RFS of} Patients with KIRC

By constructing survival curve, we continued to evaluate the effect of CREBBP expression on the patient's RFS. Our results showed that low CREBBP expression shortened the patient's RFS $(p=0.0018$, Figure 4). Subsequently, sub-groups analysis revealed that low CREBBP expression was positively correlated with poor RFS in male patients $(\mathrm{P}=0.022)$. This phenomenon also applied to G3/G4/GX ( $\mathrm{P}=0.004)$, stage III/IV ( $\mathrm{P}=0.045), \mathrm{N} 1 / \mathrm{NX}(\mathrm{P}=0.016)$ and M0 $(\mathrm{P}=0.020)$. In addition, multivariate regression analysis showed that low CREBBP expression predicted poor RFS of patients with $\operatorname{KIRC}(\mathrm{P}=0.039$; Table 2$)$. 
Table 2: Univariate and Multivariate Analysis of Relapse-Free Survival in Patients with KIRC.

\begin{tabular}{|l|c|c|c|c|c|c|}
\hline & \multicolumn{3}{|c|}{ Univariate analysis } & \multicolumn{3}{c|}{ Multivariate analysis } \\
\hline & Hazard Ratio & CI95 & Pvalue & Hazard Ratio & CI95 & Pvalue \\
\hline Age & 1.36 & $0.95-1.96$ & 0.096 & & & \\
\hline Gender & 0.77 & $0.54-1.10$ & 0.155 & & & \\
\hline Histologic grade & 1.00 & $0.66-1.39$ & 0.999 & & & \\
\hline Pathologic stage & 2.42 & $2.07-2.83$ & 0.001 & 2.57 & $1.75-3.78$ & 0.001 \\
\hline M classification & 3.42 & $2.69-4.34$ & 0.001 & 1.24 & $0.74-2.07$ & 0.409 \\
\hline N classification & 1.03 & $0.87-1.22$ & 0.730 & & & \\
\hline T classification & 2.34 & $1.94-2.83$ & 0.001 & 0.79 & $0.54-1.15$ & 0.216 \\
\hline CREBBP & 1.72 & $1.22-2.43$ & 0.002 & 1.31 & $1.13-2.03$ & 0.039 \\
\hline
\end{tabular}
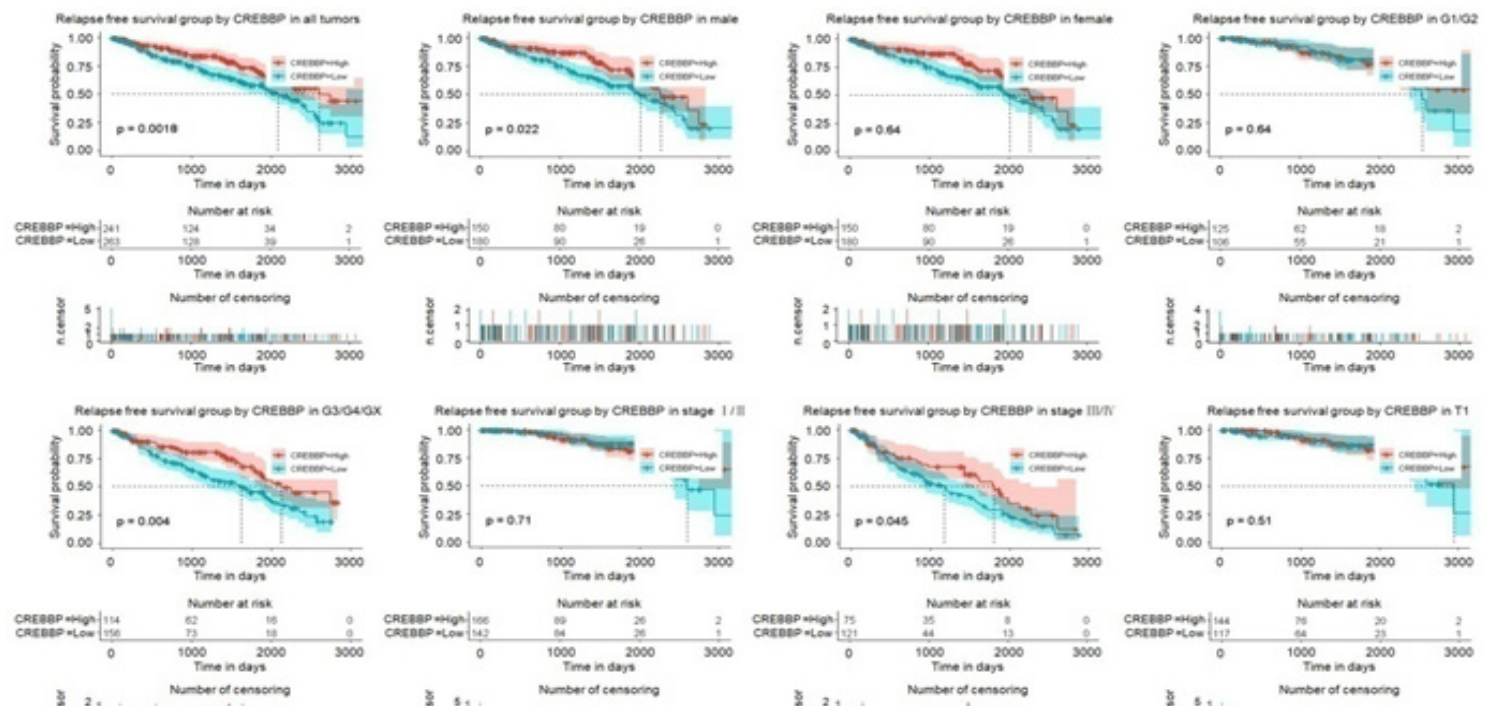

है

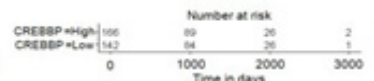

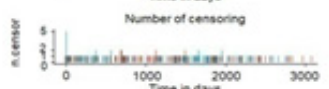

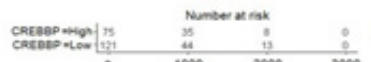

है î.
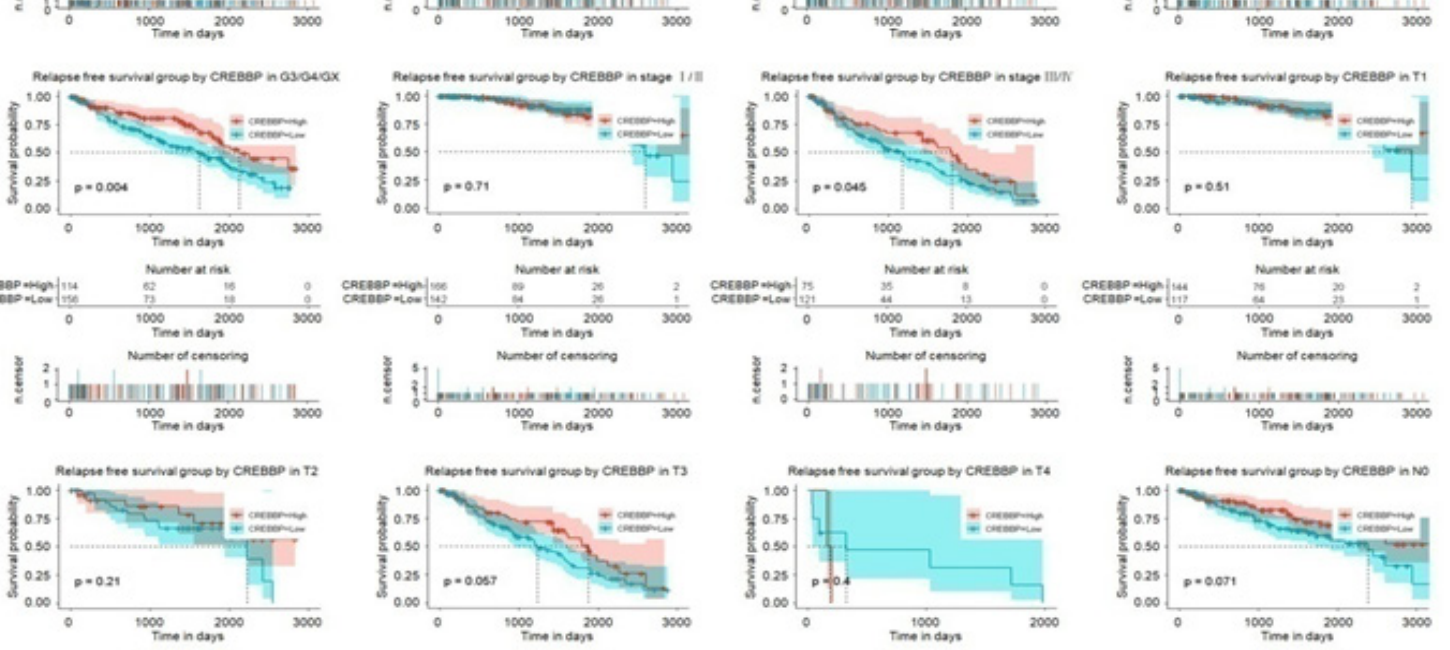

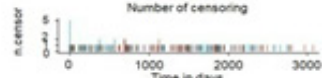
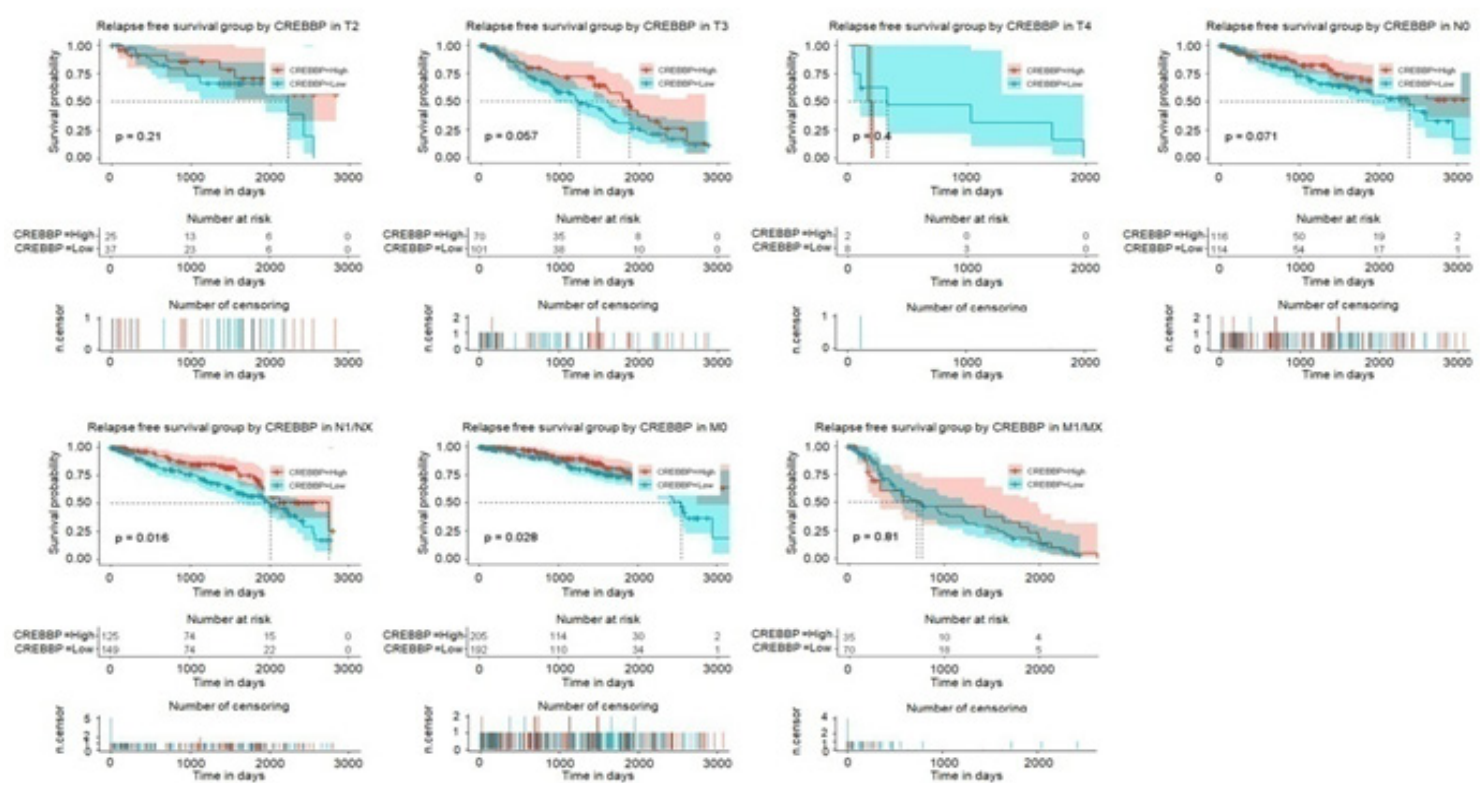

Figure 4: The Effect of CREBBP Expression on RFS in KIRC. 
Kaplan-Meier curves of CREBBP expression in all patients and in subgroup.

\section{Enrichment Analysis of Signal Pathways Related to Low CREBBP Expression}

We previously showed that low CREBBP expression was a risk predictor of OS and RFS in KIRC patients. To assess the signaling pathways activated by this gene in KIRC, GSEA was performed. We revealed signaling pathways that were significantly enriched in the low CREBBP expression phenotype, including the reactive oxygen species pathway, DNA repair and Myc-targets V2 (Table S3, Figure S1).
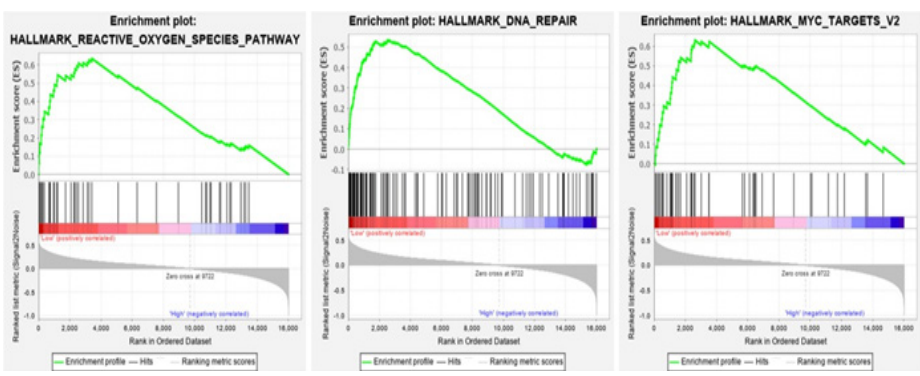

Figure 5: Gene-Set Enrichment Analysis.

GSEA results showing differential enrichment of genes related to the reactive oxygen species pathway, DNA repair and MYCtargets V2 signaling in KIRC cases with low CREBBP expression.

\section{Discussion}

By analyzing the potential relationship between the expressions of CREBBP and the clinical characteristics of KIRC patients, we demonstrated its diagnostic value, and revealed that low CREBBP expression significantly reduced the OS and RFS of patients. In addition, regression analysis indicated that CREBBP might be a useful biomarker for the prognosis of patients with KIRC.

As an important transcription co-regulator, CREBBP played important roles in angiogenesis, immune response and metabolic regulation [28,29]. In addition to histone acetyltransferase activity, the protein CREBBP encoded by this gene also had scaffolding function to stabilize the interaction of other proteins with transcription complexes [30]. Studies had shown that CREBBP mediated $\mathrm{N}$-glycosylation to change the conformation of interacting proteins, thereby regulating gene expression, cell proliferation and metabolic imbalance [31]. In this study, our results showed that CREBBP expression gradually decreased as increasing of tumor grade (G1-GX), stage (I- IV) and T-classification (T1-T4) in KIRC patients. These findings indicated that the gene played important functions in the process of tumors proliferation and metastasis. Therefore, understanding the biology of CREBBP and the correlation of its expression with the clinical characteristics of patients with KIRC might provide a new perspective for the occurrence and development of tumors. This was important to prognosis monitoring of patients, and might eventually reveal other methods to achieve effective treatments for KIRC.

CREBBP also had other functions, which might play important roles in tumor progression. It had been reported that it activated the TGF-beta signaling pathway by regulating the transcriptional activity of SMAD4, thereby affecting tumor cells proliferation and apoptosis [32]. Additionally, CREBBP was not only a transcriptional activator of the circadian rhythm, but also promoted the degradation of PCNA during nucleotide excision repair (NER) [33]. Interestingly, it caused the initiation of colorectal cancer $(\mathrm{CRC})$ through regulating the $\mathrm{Wnt} / \beta$-catenin signaling pathway, which was a main pathway for the occurrence and development of human malignant tumors $[34,35]$. Indeed, our results showed that Myc-target V2 and DNA repair signaling were significantly enriched in the low CREBBP expression phenotype, which indicated that CREBBP was likely to regulate tumorigenesis and development through the above-mentioned pathways, but the specific mechanism needed further research. Importantly, the ROC curve meant that CREBBP was a potential diagnostic-marker in KIRC.

Studies had shown that CREBBP expression was significantly higher in ovarian cancer than in normal tissues, and its expression was related to cancer stage and lymphatic metastasis [36]. In colorectal cancer, its expression was closely related to the classification and staging of tumor tissues [37]. Furthermore, the abnormal expression (mutation or deletion) of CREBBP promoted the progression of lung cancer, which indicated that the genetic changes of CREBBP might be related to the occurrence or development of malignant tumors [38]. Contrary to previous findings, we showed that CREBBP expression in KIRC was significantly lower than in normal tissues, which suggested that CREBBP expression had tissue specificity. In addition, we also found that its expression significantly reduced the OS and RFS of patients with KIRC, and it was a risk factor for poor prognosis. These findings indicated that CREBBP might be a novel molecular marker for assisting diagnosis and prognostic evaluation of KIRC.

Based on the TCGA-KIRC clinical data-set, we immediately revealed the importance of CREBBP expression in the clinical characteristics, diagnostic value and prognostic evaluation. We found that its expression not only had good clinical diagnostic value, but also had important roles in predicting the poor prognosis of patients. This was important for the treatment selection and risk evaluation of patients with KIRC.

\section{Acknowledgments}

Thanks to Dr. Lichun Wang from Sichuan University of Science \& Engineering for the R technology analysis.

\section{Funding}

This work was supported by grants from the National Key Research and Development Program of China (2017YFD0500105)

\section{References}

1. Badal SAM, Aiken WD, Chin SN (2017) Molecular Targets and Angiogenesis in Renal Cell Carcinoma, A Multitarget Approach: Mini Review. Curr Drug Targets 18: 1204-1213.

2. Tippu Z, Au L, Turajlic S (2021) Evolution of Renal Cell Carcinoma. Eur Urol Focus 7: 148-151.

3. Perry A, Lynch RM, Rusyn I, David W Threadgill (2019) Long-Term Combinatorial Exposure to Trichloroethylene and Inorganic Arsenic in Genetically Heterogeneous Mice Results in Renal Tubular Damage and Cancer-Associated Molecular 
Changes. G3 (Bethesda) 9: 1729-1737.

4. Shapira Y, Hadelsberg UP, Kanner AA, Zvi Ram, Jonathan Roth (2014) The ventricular system and choroid plexus as a primary site for renal cell carcinoma metastasis. Acta Neurochir (Wien) 156: 1469-1474.

5. Tsimafeyeu I, Zolotareva T, Varlamov S, Ruslan Zukov, Vladislav Petkau, et al. (2017) Five-year Survival of Patients with Metastatic Renal Cell Carcinoma in the Russian Federation: Results from the RENSUR5 Registry. Clin Genitourin Cancer 15: e1069-e1072.

6. Barnes CE, English DM, Cowley SM (2019) Acetylation \& Co: an expanding repertoire of histone acylations regulates chromatin and transcription. Essays Biochem 63: 97-107.

7. Huang J, Berger SL (2008) The emerging field of dynamic lysine methylation of non-histone proteins. Curr Opin Genet Dev 18: 152-158.

8. Pickart CM (2001) Mechanisms underlying ubiquitination. Annu Rev Biochem 70: 503-533.

9. Yang XJ, Seto E (2008) Lysine acetylation: codified crosstalk with other posttranslational modifications. Mol Cell 31: 449461 .

10. Audia JE, Campbell RM (2016) Histone Modifications and Cancer. Cold Spring Harb Perspect Biol 8: a019521.

11. Schizas D, Mastoraki A, Naar L, Eleftherios Spartalis, Diamantis I Tsilimigras, et al. (2018) Concept of histone deacetylases in cancer: Reflections on esophageal carcinogenesis and treatment. World J Gastroenterol 24: 4635-4642.

12. Qin J, Wen B, Liang Y (2020) Histone Modifications and their Role in Colorectal Cancer (Review). Pathol Oncol Res 26: 2023-2033.

13. Calcagno DQ, Wisnieski F, Mota ERDS (2019) Role of histone acetylation in gastric cancer: implications of dietetic compounds and clinical perspectives. Epigenomics 11: 349362 .

14. Zhao J, Gray SG, Greene CM (2019) Unmasking the pathological and therapeutic potential of histone deacetylases for liver cancer. Expert Rev Gastroenterol Hepatol 13: 247256.

15. Chen Q, Dowhan DH, Liang D (2002) CREB-binding protein/ p300 co-activation of crystallin gene expression. J Biol Chem 277: 24081-24089.

16. Frangioni JV, LaRiccia LM, Cantley LC (2000) Minimal activators that bind to the KIX domain of p300/CBP identified by phage display screening. Nat Biotechnol 18: 1080-1085.

17. Liu ST, Lu GY, Hsu YJ, Li-Chien Chang, Ching-Liang Ho, et al. (2013) Dual roles for lysine 490 of promyelocytic leukemia protein in the transactivation of glucocorticoid receptor-interacting protein 1. Biochim Biophys Acta 1833: 1799-1810.

18. Hashwah H, Schmid CA, Kasser S, Katrin Bertram, Anna Stelling, et al. (2017) Inactivation of CREBBP expands the germinal center B cell compartment, down-regulates MHCII expression and promotes DLBCL growth. Proc Natl Acad Sci U S A 114: 9701-9706.

19. Svensson K, LaBarge SA, Sathe A, Vitor F Martins, Shahriar Tahvilian, et al. (2020) p300 and cAMP response element-binding protein-binding protein in skeletal muscle homeostasis, contractile function, and survival. J Cachexia
Sarcopenia Muscle 11: 464-477.

20. Gui Y, Guo G, Huang Y, Xueda Hu, Aifa Tang, et al. (2011) Frequent mutations of chromatin remodeling genes in transitional cell carcinoma of the bladder. Nat Genet 43: 875878.

21. Korzus E (2017) Rubinstein-Taybi Syndrome and Epigenetic Alterations. Adv Exp Med Biol 978: 39-62.

22. Liu K, Wang JF, Zhan Y, Da-Lu Kong, Cui Wang (2021) Prognosis model of colorectal cancer patients based on NOTCH3, KMT2C, and CREBBP mutations. J Gastrointest Oncol 12: 79-88.

23. Ednersson SB, Stern M, Fagman H, Herman Nilsson-Ehle, Sverker Hasselblom, et al. (2020) TBLR1 and CREBBP as potential novel prognostic immunohistochemical biomarkers in diffuse large B-cell lymphoma. Leuk Lymphoma 61: 25952604.

24. Teng M, Love MI, Davis CA, Sarah Djebali, Alexander Dobin, et al. (2016) A benchmark for RNA-seq quantification pipelines. Genome Biol 17: 74.

25. Subramanian A, Tamayo P, Mootha VK, Sayan Mukherjee, Benjamin L Ebert, et al. (2005) Gene set enrichment analysis: a knowledge-based approach for interpreting genome-wide expression profiles. Proc Natl Acad Sci USA 102: $15545-$ 15550.

26. Robin X, Turck N, Hainard A, Natalia Tiberti, Frédérique Lisacek, et al. (2011) pROC: an open-source package for R and $\mathrm{S}+$ to analyze and compare ROC curves. BMC Bioinformatics 12:77.

27. Siena S, Sartore-Bianchi A, Di Nicolantonio F, Julia Balfour, Alberto Bardelli, et al. (2009) Biomarkers predicting clinical outcome of epidermal growth factor receptor-targeted therapy in metastatic colorectal cancer. J Natl Cancer Inst 101: 13081324.

28. Höpken UE (2017) Targeting HDAC3 in CREBBPMutant Lymphomas Counterstrikes Unopposed Enhancer Deacetylation of B-cell Signaling and Immune Response Genes. Cancer Discov 7: 14-16.

29. Huang L, Liu D, Wang N, Shaoping Ling, Yuting Tang, et al. (2018) Integrated genomic analysis identifies deregulated JAK/STAT-MYC-biosynthesis axis in aggressive NK-cell leukemia. Cell Res 28: 172-186.

30. Ogryzko VV, Schiltz RL, Russanova V, B H Howard, Y Nakatani, et al. (1996) The transcriptional coactivators p300 and CBP are histone acetyltransferases. Cell 87: 953-959.

31. Siddique H, Rao VN, Reddy ES (2009) CBP-mediated posttranslational N-glycosylation of BRCA2. Int J Oncol 35: 387 391.

32. Zhao T, Satou Y, Sugata K, Paola Miyazato, Patrick L Green, et al. (2011) HTLV-1 bZIP factor enhances TGF- $\beta$ signaling through p300 coactivator. Blood 118: 1865-1876.

33. Cazzalini O, Sommatis S, Tillhon M, Ilaria Dutto, Angela Bachi, et al. (2014) CBP and p300 acetylate PCNA to link its degradation with nucleotide excision repair synthesis. Nucleic Acids Res 42: 8433-8448.

34. Tian X, Sun D, Zhao S, Hua Xiong, JingYuan Fang (2015) Screening of potential diagnostic markers and therapeutic targets against colorectal cancer. Onco Targets Ther 8: 16911699.

35. Zhu J, Zhang S, Gu L, Wen Di (2012) Epigenetic silencing of 
DKK2 and Wnt signal pathway components in human ovarian carcinoma. Carcinogenesis 33: 2334-2343.

36. Gong S, Chen Y, Meng F, Yadi Zhang, Chanyuan Li, et al. (2018) Roflumilast enhances cisplatin-sensitivity and reverses cisplatin-resistance of ovarian cancer cells via cAMP/PKA/ CREB-FtMt signalling axis. Cell Prolif 51: e12474.

37. Ishihama K, Yamakawa M, Semba S, Hiroaki Takeda, Sumio
Kawata, et al. (2007) Expression of HDAC1 and CBP/p300 in human colorectal carcinomas. J Clin Pathol 60: 1205-1210.

38. Horton SJ, Giotopoulos G, Yun H, Shabana Vohra, Olivia Sheppard, et al. (2017) Early loss of Crebbp confers malignant stem cell properties on lymphoid progenitors. Nat Cell Biol 19: 1093-1104.

Copyright: (C2021 Chaoxiang Lv and Tiecheng Wang, et al. This is an openaccess article distributed under the terms of the Creative Commons Attribution License, which permits unrestricted use, distribution, and reproduction in any medium, provided the original author and source are credited. 